\title{
Validation of SPHC and CRASH codes in modeling of linear and non-linear Richtmyer-Meshkov instabilities
}

\author{
Stanic, M. and Cassibry, J.T. \\ University of Alabama in Huntsville, Huntsville, AL, 35899 \\ Stellingwerf, R. F. \\ Stellingwerf Consulting, Huntsville, AL, 35803 \\ Chou, C-C. and Fryxell, B.J. \\ University of Michigan, Ann Arbor, MI, 48109 \\ Abarzhi, S.I. \\ The University of Chicago, Chicago, IL, 60637
}

\begin{abstract}
Richtmyer-Meshkov instability (RMI) plays an important role in a broad variety of phenomena in nature and technology and is of special interest in the fields of shockturbulence interaction, supersonic aerodynamic flows, and inertial and magneto-inertial fusion. The instability develops when a shock refracts an interface between two fluids with different values of the acoustic impedance, and RMI dynamics is defined primarily by the flow Mach number and the Atwood number for the two fluids. For continuous fluid dynamic (CFD) codes, numerical modeling of RMI is a severe task, which imposes high requirements on the resolution, accuracy and spatio-temporal dynamic range of the simulations. Modeling of high-Atwood and high-Mach flows, which are of interest in practical applications, is even more challenging, as it requires shock capturing, interface tracking and accurate accounting for the dissipation processes. We used Smooth Particle Hydrodynamics Code (SPHC) and Center for RAdiative Shock Hydrodynamics (CRASH) codes to mutually evaluate the codes and compare their results against the analytical RMI theory. The numerical and theoretical results are in good qualitative and quantitative agreement with one another. These results indicate that at large scales the nonlinear dynamics of RMI is a multi-scale processes; at small scale the flow field is heterogeneous and is characterized by appearance of local microscopic structures; the coupling between the scales has a complicated character.
\end{abstract}

\section{Introduction}

Shock-turbulence interaction plays a key role in high speed aerodynamic flows [1-3]. Its accurate quantification is important for practical applications and flow control in supersonic vehicles, scramjet combustors, and film-cooled turbine blades [3]. Shocks inevitably occur when the flow speed increases to the speed of sound. The shock enhances turbulence production, substantially modifies its length scale and dissipation properties, and may lead to flow separation and unsteadiness [1-3]. In a coarse approximation, the amplification of the turbulence production by a shock wave can be obtained from the Rankine-Hugoniot condition and from the decomposition of turbulent fluctuations into the uncoupled acoustic, vorticity and entropy modes [4-9]. To describe the shock-turbulence interactions at fine scales, a better understanding is required of the influence of the shock on the turbulent vorticity fields, in particular on the vortex stretching, tilting and dilatation, and on the baroclinic vorticity production [1,10]. The baroclinic vorticity is produced whenever pressure and density gradients are pointed in opposite directions [11]. It couples the velocity and density fields and results in extensive interfacial turbulent mixing [12, 13]. The mixing

American Institute of Aeronautics and Astronautics 
induces strong interplay of the acoustic, vorticity and entropy modes, and governs the fluctuations of the pressure and temperature fields [14]. Its properties depart from the scenario of canonical turbulence flows [12-16].

To capture the baroclinic vorticity production and the induced turbulent mixing, one should accurately account for the evolution of the unstable interfaces, which approximate the regions with sharp changes of vector and scalar fields of the turbulent flow, and to apply a so-called sharp boundary approximation [13, 14]. Traditional methods employed in the studies of shock-turbulence interaction consider the flow dynamics in a 'diffusive' approximation, and for these methods to capture the baroclinic vorticity production is a challenge [17-19].

A representative example of the baroclinically unstable flow is the Richtmyer-Meshkov instability (RMI) [2022]. RMI develops when a shock wave refracts the interface between two homogeneous fluids with different values of the acoustic impedance [20-23]. Richtmyer-Meshkov (RM) turbulent mixing plays an important role in many natural and technological processes, such as inertial confinement and magneto-inertial fusion, core-collapse supernova, impact dynamics of liquids, and supersonic combustion [12,13,23-25]. For instance, to achieve magnetoinertial fusion [26-28], magnetized target plasma is compressed by imploding plasma liner. The liner is formed from an array of converging high Mach number high-velocity plasma jets, which are generated by pulsed plasma guns. During the implosion process, the plasma may become Richtmyer-Meshkov unstable, and RM mixing may significantly influence the liner stability, the interaction between the liner and the target, and the fusion [26-28].

Mitigation and control of RMI is a long-standing problem [20-22]. The fundamental issues to be understood are the evolution and coupling of the large-scale coherent structures and the small-scale structures characterizing RM turbulent mixing [12,13]. Our work studies nonlinear RMI for high Mach number flows and for fluids with contrasting fluid densities, when the effect of the baroclinic vorticity production by the shock wave is the most pronounced [22-24,29-31]. In our simulations the Mach number ranges from 3 to 10, the Atwood number $A=\left(\rho_{h}-\rho_{l}\right) /\left(\rho_{h}+\rho_{l}\right)$ ranges from 0.6 to 0.95 , where $\rho_{h(l)}$ is the density of the heavy (light) gas, and the ratio between the initial amplitude and period of the large-scale coherent structure ranges from 0.06 to 0.25 .

Our research is driven by the theoretical analysis $[12,13,30]$ and employs the Smoothed Particle Hydrodynamics (SPHC code) and Continuous Fluid Dynamics (CRASH code) numerical methods. By the authors' knowledge, this is one of the first explorations of the extreme parameter regime, in which we attempt to effectively synergize the advantages of the continuous dynamics and the kinetic dynamic approximations with the rigor of theoretical studies [32]. The theoretical analysis serves to suggest for the numerical modeling the reliable metrics of the flow, and to identify new properties of the dynamics as observed in the simulations [12,13]. The advantages of the particle method is that it enables accurate capturing the effect of microscopic processes on the large-scale dynamics, and self-consistently accounts for the heterogeneous structures of the flow field as well as the non-equilibrium transports. The advantages of the continuous approximation are that it can model the nonlinear dynamics spanning significant range of scales and approach the time-scales of interest to the experiments and practical applications.

Our results indicate that: (i) The nonlinear evolution of the large-scale coherent dynamics in RichtmyerMeshkov mixing is an essentially non-local and multi-scale processes. (ii) At late stages of flow evolution, the dynamics at small-scale is heterogeneous, and is characterized by the appearance of local microscopic structures, such as reverse jets. (iii) The coupling between the large and small scales has a complicated character, and departs from the consideration proposed by simple heuristic models [33].

\section{Outline of evolution of the Richtmyer-Meshkov instability and theoretical analysis}

According to the decades-long history of studies, the Richtmyer-Meshkov instability evolves as follows $[12,13,20-22,29-40]$. RMI develops when a shock wave refracts through a perturbed interface, whether it propagates from the light fluid to the heavy gas or in the opposite direction $[20,21]$. The interaction of the shock wave with the interface results in a complicated dynamics, which can be presented as a superposition of two motions. On the one hand, the interface moves as a whole with a velocity, at which it would move if it would be ideally planar. The velocity of this motion can be derived from one-dimensional calculations and it is an order of magnitude less than the speed of sound $[20,21,30]$. On the other hand, small perturbations of the velocity and density fields at the interface start to grow [20,21,29-40]. The growth is due to the 'impulsive' acceleration of the heavy gas by the light gas, and the growth-rate value is determined by the baroclinic vorticity, which is induced by the shock [20,21,29].

For small amplitude initial perturbation, an adequate analytical description of the linear regime of RMI was found recently in Ref.[29]. This theory punctually derives the growth-rate of RMI in cases of strong and weak 
shocks for gases with similar and contrasting densities. For small but finite amplitude of the initial perturbation, corrections for the growth-rate value can be found within the frames of the weakly nonlinear analysis [36-38]. Overall, in the linear regime of RMI the value of the growth-rate is two orders of magnitude less than the speed of sound [30].

In the nonlinear regime of RMI the growth-rate decreases and a large-scale coherent structure of bubbles and spikes appears: The light (heavy) fluid penetrates the heavy (light) fluid in bubbles (spikes) $[12,13]$. The spatial period of the structure is set by the initial perturbation or by the mode of fastest growth, and the amplitude is defined by the bubble and spike positions [12,13]. Shear-driven Kelvin-Helmholtz instabilities produce small-scale structures on the sides of evolving spikes [29-40]. Roll-up of vortices causes a mushroom-typed shape of the spike [29-40]. Singular and non-local aspects of the interface evolution make the nonlinear dynamics of RMI a very complicated theoretical problem [12,13].

An adequate analytical description of the nonlinear RMI was found recently in Refs. [12,13,30,39,40]. The nonlinear analysis $[12,30]$ employed group theory and found that the late-time dynamics of RMI is a multi-scale process, which is governed by two independent length scales: the spatial period and the amplitude of the front. For the bubble front the nonlinear theory suggests that after a short stage of the shock-interface interaction, the bubble curvature and velocity change linearly with time; then, in the weakly non-linear regime, the curvature reaches an extreme value, dependent on the initial conditions and the Atwood number; asymptotically, the bubble flattens and decelerates $[12,13,39,40]$. The flattening of the bubble front is a distinct feature of RMI universal for all values of the Atwood number, and it is a qualitative indicator of the multi-scale character of the dynamics $[12,13]$.

Eventually a mixing zone develops, and in the chaotic regime the bubbles and spikes decelerate, the energy, which is induced by the initial shock, gradually dissipates in small-scale structures, and the disordered turbulent fluctuations decays with time [29-40]. At this stage, viscosity may start to play a dominant role. RM mixing is a non-local, inhomogeneous and statistically unsteady turbulent process, and its properties depart from the scenario of canonical turbulent flows [13-16].

Theoretical analysis $[12,13,30,40]$ emphasizes the necessity to characterize the front dynamics with high accuracy and precision enabling the derivation of higher order spatial and temporal derivatives, to conduct the observations of the robust macroscopic parameters over significant dynamic range, and to tightly control the experimental parameters, such as Mach and Atwood numbers (see Ref.[41] for more details). The analysis $[12,13,30]$ also indicates that the microscopic processes in RM mixing flow may significantly influence the macroscopic dynamics, and that the coupling between the large and small scales has a complicated character $[32,42]$.

To adequately describe the mixing process and to provide an insight for the problem of shock-turbulence interactions, we perform a comparative numerical modeling study, which synergizes the advantages of the continuous dynamics and the kinetic dynamic approximations. We will explore the parameter regime, in which the extreme properties of the material mixing are most pronounced, and which include strong shock, strong gradients of density and pressure, inherently non-equilibrium dynamics, and non-local interactions among the many scales.

\section{Code principles and test cases}

\section{A. SPHC theoretical basis}

Smoothed Particle Hydrodynamics (SPH) is a Lagrangian method in computational fluid dynamics [43] that allows relatively easy treatment of the governing equations of the fluid motion. Finite difference methods approximate partial differential equations by approximating gradients of field variables located at a finite number of points in space using differencing methods. Similarly, SPH makes the kernel and particle approximations to reduce the governing equations to a set of ordinary differential equations. In particular, any field variable can be expressed as

$$
f(\vec{x})=\int_{\Omega} f\left(\vec{x}^{\prime}\right) \delta\left(\vec{x}-\vec{x}^{\prime}\right) d \vec{x}^{\prime}
$$

where $\boldsymbol{x}$ 's are the position vectors of the particles, $\Omega$ the integral volume that contains $\boldsymbol{x}$ and $\delta$ as Dirac delta function. In the kernel approximation, the Dirac delta function is then replaced by a so-called kernel function $W$ with following properties: 

1) $\int_{\Omega} W\left(\vec{x}-\vec{x}^{\prime}, h\right) d \vec{x}^{\prime}=1$
2) $\lim _{h \rightarrow 0} W\left(\vec{x}-\vec{x}^{\prime}, h\right)=\delta\left(\vec{x}-\vec{x}^{\prime}\right)$
3) $W\left(\vec{x}-\vec{x}^{\prime}, h\right)=0$, when $\left|\vec{x}-\vec{x}^{\prime}\right| \geq k h$

where $h$ is referred to as the smoothing length, and $k$ is a coefficient which determines the support domain boundary (see [43-48] for details). Substitution of the kernel function for the Dirac delta function, we obtain

$$
<f(\vec{x})>=\int_{\Omega} f\left(\vec{x}^{\prime}\right) W\left(\vec{x}-\vec{x}^{\prime}, h\right) d \vec{x}^{\prime}
$$

The brackets around $f(\boldsymbol{x})$ represent the Kernel Approximation Operator (KAO). When the spatial derivative operator $\nabla$ is applied to KAO, the kernel function multiplier on the right hand side becomes a gradient of the kernel function, leaving the initial function $f(x)$ intact.

$$
<\nabla \cdot f(\vec{x})>=-\int_{\Omega} f\left(\vec{x}^{\prime}\right) \nabla W\left(\vec{x}-\vec{x}^{\prime}, h\right) d \vec{x}^{\prime}
$$

This is very convenient as it simplifies the implementation of the method. Once this approach is discretized (particle approximation), very simple expressions can be obtained in form of Eq. (4) and Eq. (5):

$$
\begin{gathered}
<f(\vec{x})>\approx \sum_{j=1}^{N} \frac{m_{j}}{\rho_{j}} f(\vec{x}) W\left(\vec{x}-\vec{x}_{j}, h\right) \\
<\nabla \cdot f(\vec{x})>\approx-\sum_{j=1}^{N} \frac{m_{j}}{\rho_{j}} f\left(\vec{x}_{j}\right) \nabla W\left(\vec{x}-\vec{x}_{j}, h\right)
\end{gathered}
$$

For brevity, detailed derivations of equations of motion will not be presented here. For further details, see, e.g. references [43-48].

As any other method for solving the fluid equations of motion, the SPH has some limitations. For instance, certain efforts have to be taken in order to find an efficient algorithm for "neighbor" particle seeking as well as for overcoming of particle-penetration problems by implementing artificial viscosity. These purely numerical issues are mostly relying on programmers experience and on their empirical knowledge. In this sense SPHC is one of the more robust SPH codes, as it has been developing continuously for more than twenty years, and therefore is a good candidate for simulations of highly non-linear dynamics of the Richtmyer-Meshkov instability.

\section{B. CRASH code theoretical basis}

For the CFD simulation we used an Eulerian code package, Center for Radiative Shock Hydrodynamics (CRASH) code. Extended from BATS-R-US (Block-Adaptive Tree Solar wind Roe Upwind Scheme), CRASH is capable of 3D simulation with multi-group radiation diffusion model and adaptive mesh refinement. For more details on CRASH's numerical method and current capabilities, please see [49]. For purposes of present work we only use its hydrodynamics simulation on a fixed, 2D Cartesian grid.

We employed the CRASH's implementation of the HLLE [49] scheme, which is second order in both space and time. Explicit 2-stage Runge-Kutta scheme with CFL number 0.8 is used for time integration, and the flux is calculated with the reconstructed linear slopes of the state variables within the cells using generalized Koren's limiter. In more detail, a flux limiter is required for these Riemann solvers to extrapolate the state variable $U$ from the left and right cell centers to the cell face at $i=1 / 2$ as 


$$
\begin{gathered}
U_{i+1 / 2}^{L}=U_{i}+\frac{1}{2} \bar{\Delta} U_{i} \\
U_{i+1 / 2}^{R}=U_{i+1}-\frac{1}{2} \bar{\Delta} U_{i+1}
\end{gathered}
$$

where $\bar{\Delta} U_{i}$ is the limited slope in cell $i$. For the generalized Koren's limiter, the limited slopes in the left and right extrapolations are constructed asymmetrically as

$$
\begin{aligned}
& \bar{\Delta}^{L} U_{i}=\min \bmod \left[\beta\left(U_{i+1}-U_{i}\right), \beta\left(U_{i}-U_{i-1}\right), \frac{2 U_{i+1}-U_{i}-U_{i-1}}{3}\right] \\
& \bar{\Delta}^{R} U_{i}=\min \bmod \left[\beta\left(U_{i+1}-U_{i}\right), \beta\left(U_{i}-U_{i-1}\right), \frac{U_{i+1}-U_{i}-2 U_{i-1}}{3}\right]
\end{aligned}
$$

where $\beta$ is an adjustable parameter which controls the amount of numerical dissipation with admissible range $1.0 \leq \beta \leq 2.0$. $\beta$ is fixed to be 2.0 for this study, and the resolution is 64 cells per wavelength. For these CFD runs, the wavelength is equal to the width of the shock tube, so the physical wavelength is also $1 \mathrm{~cm}$.

\section{SPHC test simulation of Rayleigh-Taylor instability}

In order to validate SPHC code's ability to model Richtmyer-Meshkov instability, a test case of Rayleigh-Taylor (RT) from Moylan's work [50] has been used. Moylan developed a numeric model of Rayleigh-Taylor instability for the purposes of simulating influence of shocks and supersonic flowfields on water droplets. The model was constructed and compared with the analytical theory of Chandrasekhar [51] and Joseph [52]. Two parameters were used to assess the ability of the code to model the RT instability: the wavelength of the most unstable wave, and the growth rate of that wave. Initial parameters values were surface tension for water $(72$ dyne $/ \mathrm{cm})$, density of water $(1$ $\mathrm{g} / \mathrm{cc})$, and drop acceleration $\left(1.0 \times 10^{7} \mathrm{~cm} / \mathrm{s}^{2}\right)$. The acceleration term is near that predicted by Joseph [17] $\left(6.47 \times 10^{7}\right.$ $\mathrm{cm} / \mathrm{s}^{2}$ ) for a Mach 3 demise' scenario. Joseph's theory predicts that with these parameters, maximum growth rate will occur at a wavelength of $0.029 \mathrm{~cm}$ at a growth rate of $37,873 / \mathrm{sec}$.
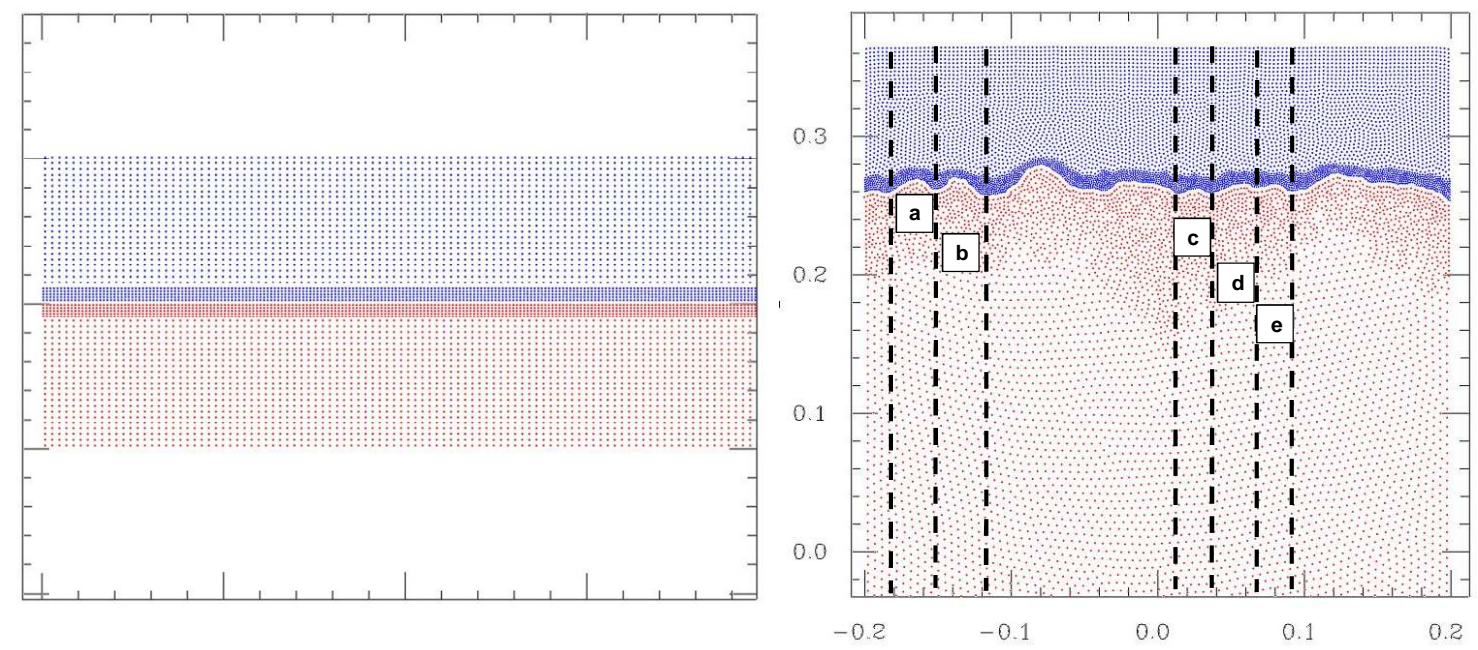

Figure 1: SPHC R-T Instability Model, $0 \mu \sec (a), 35 \mu \sec (b)$, [50]

The results of the SPHC simulations gave a modeling difference compared to theory of $5 \%$, which represents a good correlation to the theory. The resulting numerical prediction appears a function of the number of particles in the model. If particle count would be decreased by $50 \%$, the predicted most unstable wave would increase to 0.37 $\mathrm{cm}$ or $27 \%$ error. It is therefore important to account for the impact of particle resolution when discussing RT instability in SPHC. The results of the test case are presented in Table 1. The wave height and rate of growth were 
recorded in intervals of $10 \mu \mathrm{sec}$, with first $30 \mu \mathrm{sec}$ being skipped because no sufficient wave-geometry was developed.

Table 1: SHPC R-T Growth Rate Results, [50]

Therefore, the conclusion of the numerical comparisons to the 2-D planer RT theory is that the SPHC code does in fact produce sufficient accuracy. Due to physical similarity and resemblance of Richtmyer-Meshkov and RayleighTaylor instabilities, one may expect that SPHC is capable of capturing Richtmyer-Meshkov instability and providing reasonable data for further theoretical studies.

\begin{tabular}{|c|c|c|c|c|c|}
\hline Time & $\begin{array}{c}\text { Wave } \\
\text { Height }\end{array}$ & Growth & \multirow{2}{*}{$\begin{array}{c}\text { Theory } \\
\left(e^{n t}\right)\end{array}$} & \multirow{2}{*}{$\begin{array}{c}\text { SPHC } \\
\left(x_{2} / x_{1}\right)\end{array}$} & \multirow[t]{2}{*}{ \% Diff } \\
\hline usec & $(\mathrm{cm})$ & $(\mathrm{cm})$ & & & \\
\hline 30 & 0.0093 & & & & \\
\hline 40 & 0.0198 & 0.0105 & 1.46 & 2.13 & 0.69 \\
\hline 50 & 0.028 & 0.0082 & 1.46 & 1.41 & 1.03 \\
\hline 60 & 0.0384 & 0.0104 & 1.46 & 1.37 & 1.06 \\
\hline 70 & 0.0523 & 0.0139 & 1.46 & 1.36 & 1.07 \\
\hline
\end{tabular}

\section{CRASH test simulation of Rayleigh-Taylor instability}

Similarly, a resolution study of 2-D planer Rayleigh-Taylor instability was developed for CRASH. Since CRASH lacks physical model of surface tension and viscosity, we focused on early-time behavior and compared the growth rate to the prediction of the simple linear theory where the mixing zone width of the RTI grows exponentially as

$$
\text { width } \propto \exp (t \sqrt{2 \pi A g / \lambda})
$$

where $g$ is the gravitational acceleration, and $\lambda$ is the spatial period. With CRASH, we nominally set $A=1 / 3, g=$ $9806.65 \mathrm{~m} / \mathrm{s}^{2}$, and $\lambda=0.0002 \mathrm{~m}$ and initialized with a small velocity perturbation around the interface. Since the behavior of the system without viscosity doesn't depend on the length scale, we use $\lambda$ as the length-scale and $\sqrt{\lambda / 2 \pi A g}$ for the time-scale.

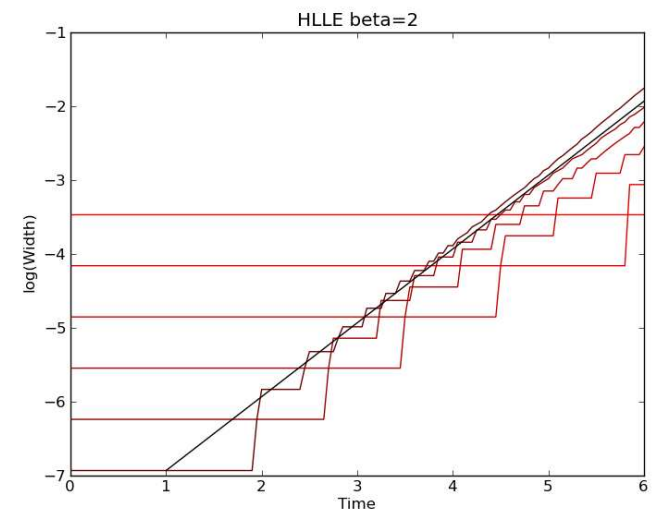

Figure 2: Mixing zone width vs. time. Darker color indicates higher resolution as number of cells per wavelength, which ranges from $2^{5}$ to $2^{10}$. The black line marks the linearized analytical result with slope $=1$.

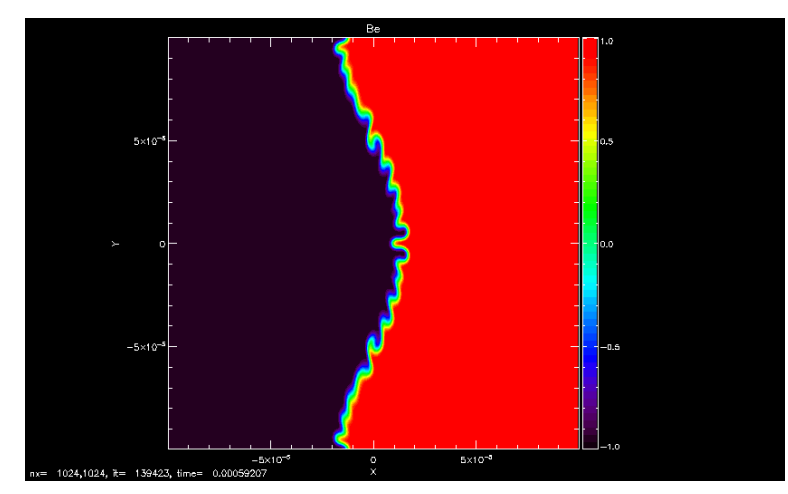

Figure 3: Level-set plot of the HLLE simulation with 1024 cells per wavelength at $t=6$ natural time unit.

As is seen from Figures 2 and 3, after the instability overcomes the numerical dissipation, its growth rate follows the linearized analytical result. It deviates again later in time for high-resolution runs. This is due to the spontaneous growth of the smaller-wavelength instabilities, whose presence is unavoidable in the inviscid simulations. 


\section{Results\& Discussion}

\section{A. SPHC results}

Total of 18 simulations have been carried out. Parameters that have been varied are Mach number (3, 5 and 10), Atwood number (0.6, 0.8 and 0.95 ) and initial size of the amplitude (0.06 and 0.25 of the wavelength).

Data has been extracted from 31 moving probes, which are essentially designated particles that record their state (flow parameters) at certain time frames and export them to the data history file. Initially all the probes are evenly distributed along the two-fluid interface. As simulations progress, due to appearance of vorticies, most probes are perturbed from their original position in the probe-row, causing mixing of the probes and destroying the opportunity to get useful data out of them. However, at places where little or no vortexing occurs (e.g. the tip of the bubble and the tip of the spike), the probes remain unperturbed and follow the flow fields. These probes where used to obtain the data throughout simulations.

Diagnostic parameters that have been obtained in the simulations include the amplitude growth, the rate of the amplitude growth, the acceleration of amplitude growth and curvature of the bubble front [12,13,30]. All of the results were analyzed using appropriate scaling factors based on the initial perturbation wavelength $\lambda$ and post-shock speed of sound in the low-density region $c$. First four parameters have been obtained by direct reading of the data from the appropriate probes that resided in the vicinity of the bubble/spike tip throughout the simulations. For the sake of avoiding excess number of figures and as the trends are similar among the simulations (with primary difference being in the length of simulation), only the amplitude growth and rate of amplitude growth for $M=10$ and $M=3$ are presented in Figures 4 through 7, respectively.

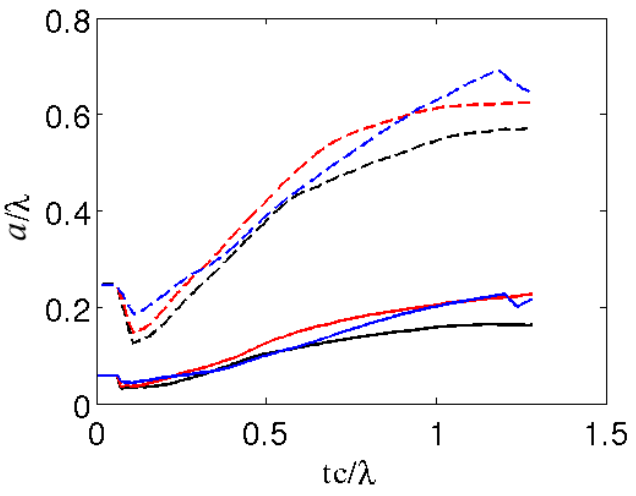

Figure 4: Scaled amplitude growth for $M=10$ cases. Solid lines represent runs with initial amplitudes of $0.06 \lambda$ and dashed lines stand for runs with initial amplitudes of $0.25 \lambda$. , red and blue colors are $A=0.6, A=0.8$ and $A=0.95$, respectively.

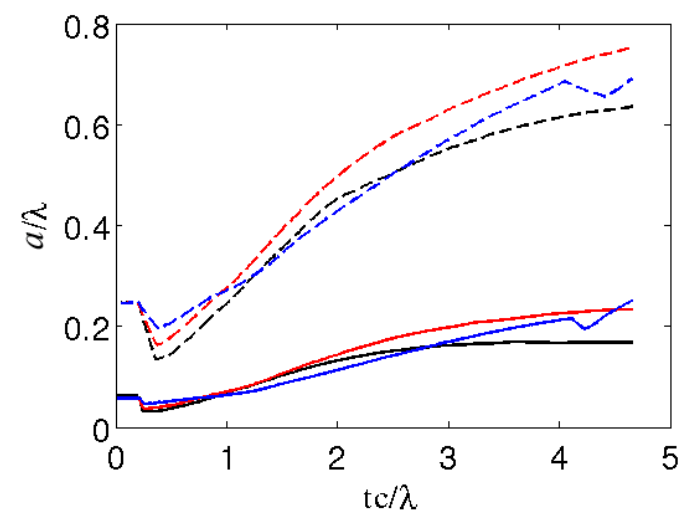

Figure 6: Scaled amplitude growth for $M=3$ cases. Solid lines represent runs with initial amplitudes of $0.06 \lambda$ and dashed lines stand for runs with initial amplitudes of $0.25 \lambda$. , red and blue colors are $A=0.6, A=0.8$ and $A=0.95$, respectively.

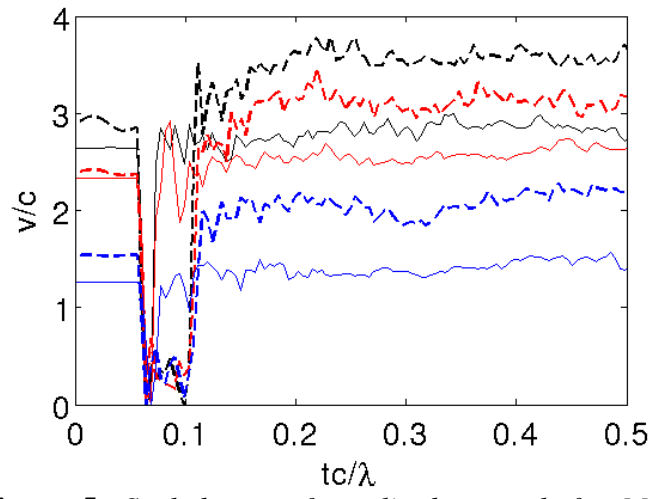

Figure 5: Scaled rate of amplitude growth for $M=10$ cases. Solid lines represent runs with initial amplitudes of $0.06 \lambda$ and dashed lines stand for runs with initial amplitudes of $0.25 \lambda$, red and blue colors are $A=0.6, A=$ 0.8 and $A=0.95$, respectively.

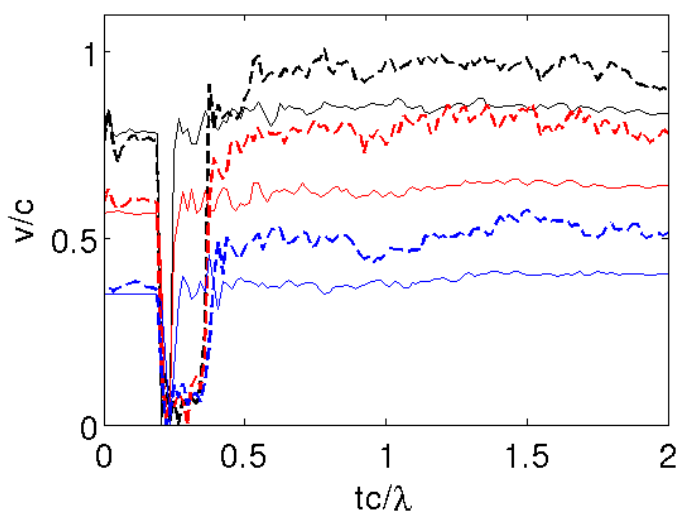

Figure 7: Scaled rate of amplitude growth for $M=3$ cases. Solid lines represent runs with initial amplitudes of $0.06 \lambda$ and dashed lines stand for runs with initial

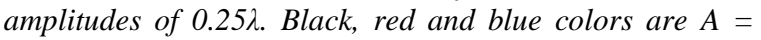
0.6, $A=0.8$ and $A=0.95$, respectively. 
In Figures 4, 5, 6 and 7 one can clearly see that both the amplitude growth and the initial rates of the amplitude growth depend strongly on the initial amplitude. On the other hand, the amplitude growth doesn't show significant dependence on the Atwood number, whereas the final value of rate of amplitude growth does seem to be strongly influenced by it.

Curvature $\zeta$ was calculated via the use of three probes at the top of the bubble, e.g. via recording their position and employing simple analytic geometry to calculate the radius of the circle whose arc passes through the three points. From there data curvature $\zeta$ is calculated as the inverse of the radius. This method of estimation curvature is not considered as an ideal [30], because the position of a single probe may significantly influence the overall result. However, our SPHC simulations did show fairly good agreement with the theoretical predictions at least qualitatively $[12,30,39,40]$ and did find that curvature of the bubbles front decreases with time. This result holds for all the simulations, as one may see in Figure 8.

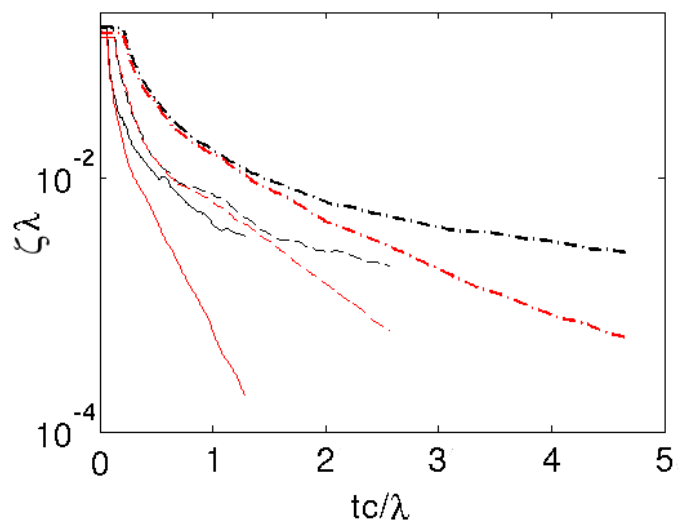

Figure 8: Logarithmic plot of scaled curvature for $A=0.6$, initial amplitude $0.06 \lambda, M=10$ runs (solid lines), $M=5$ (dashed lines) and $M=3$ (dash-dot lines). Black and red stand for initial amplitudes of $0.06 \lambda$ and $0.25 \lambda$.

Acceleration was directly recorded from the probes and has fairly uniform results across the parameter regime. Strong acceleration is experienced by the fluids as the initial shock refracts across the interface, but at later times the acceleration settles down to around zero, as shown in Figure 9.

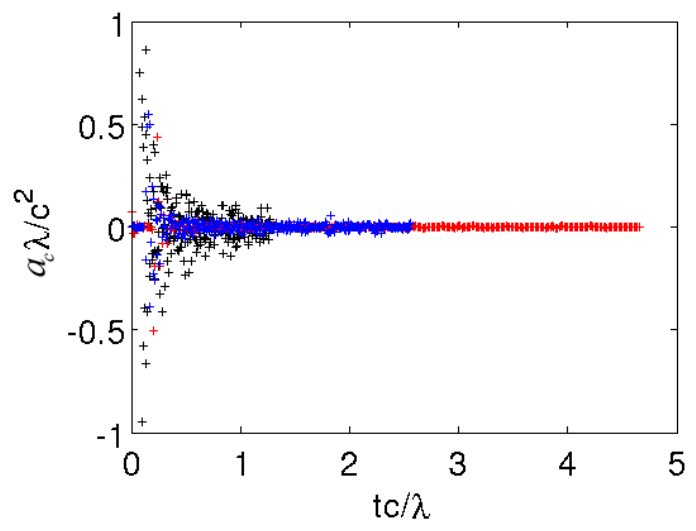

Figure 9: Plot of scaled acceleration for $A=0.6$,

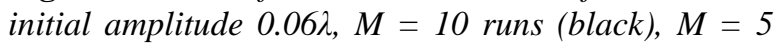
(blue) and $M=3$ (red). 
An important qualitative result is the development of the shear-driven Kelvin-Helmholtz instability at the interface, see Figure 10. The other important qualitative result that has been recorded is the appearance of the 'reverse jets' ('cumulative jets') [53]. These jets occur at late times of simulations at roots of the spikes and are propagating in the direction of the initial shock (therefore being reversed with respect to the propagation of the spike). The jets can be clearly seen when the particular plots are made, e.g. the temperature plot as in Figure 11. This phenomenon indicates that in the nonlinear regime the dynamics at small scales is heterogeneous and that the coupling between the large and small scales has a complicated character.

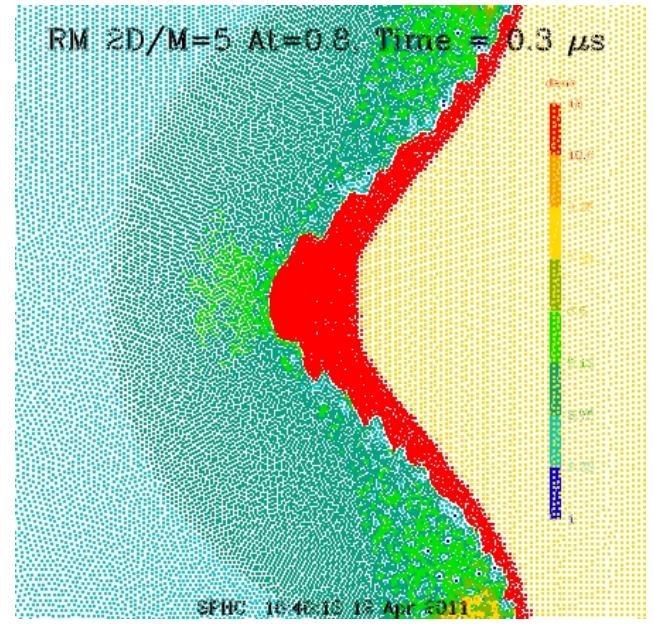

Figure 10: Kelvin-Helmholtz instability developing at the surface of the bubble.

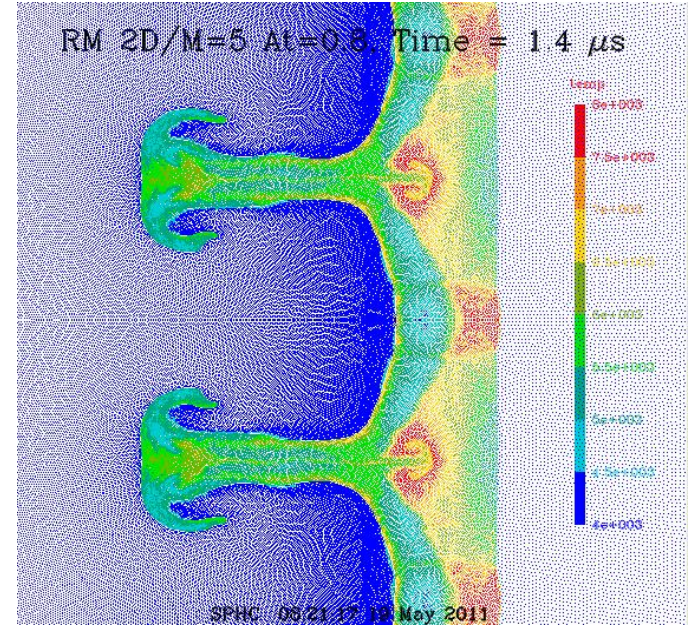

Figure 11: Temperature plot in range between $4000 \mathrm{~K}$ and $8000 \mathrm{~K}$, clearly showing "reversed jets" in the base of spikes (between bubbles).

\section{B. CRASH Results}

CRASH results qualitatively agree with SPHC results, providing mutual support between the codes. Figures 12 and 13 provide CRASH plots for amplitude growth and rate of amplitude growth for the case $M=3, A=0.6$ and initial amplitude $a=0.06 \lambda$, which are in agreement with Figures 6 and 7 of the SPHC results. In order to see the agreement it is important to have in mind that all SPHC data has been scaled and made "absolute", so the minimum $y$ value on the graphs is zero, while same has not been done for CRASH results.

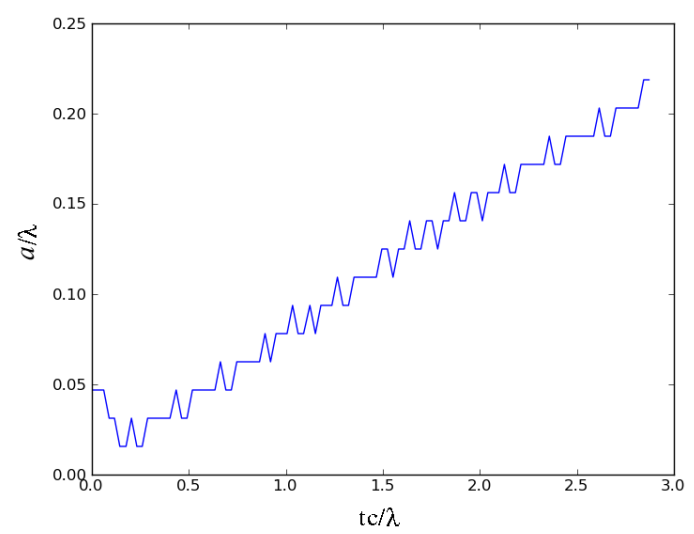

Figure 12: CRASH scaled amplitude growth for the case $M=3, A=0.6$ and initial amplitude $a=0.06 \lambda$.

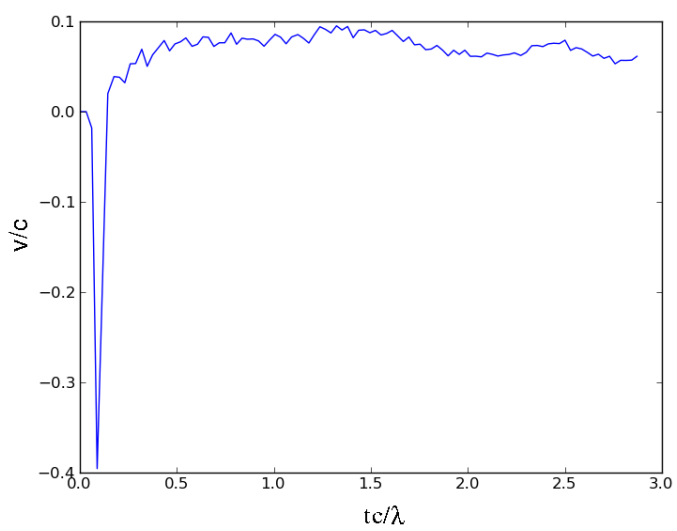

Figure 13: $C R A S H$ scaled rate of amplitude growth for the case $M=3, A=0.6$ and initial amplitude $a=$ $0.06 \lambda$.

Runs with $A=0.95$ and with small initial perturbation $(0.06 \lambda)$ exhibit apparent oscillations in the rate of the amplitude growth. The periods of the oscillations are estimated from the plots to be: $2 \mu$ s (Mach 3), $1.25 \mu$ s (Mach 
4), and $0.67 \mu$ s (Mach 10). These periods are within 33\% agreement with the time required for the sound wave to travel across the wavelength/tube width, $\lambda / c$, calculated from the post-shock density and pressure as $2.56 \mu$ s (Mach 3), $1.66 \mu$ s (Mach 5), and $0.87 \mu$ s (Mach 10) respectively. Further examination of the spike and bubble velocities indicates that they oscillate with phases opposite to each other. Qualitatively the CRASH runs show standard features of RMI evolution [29-40].

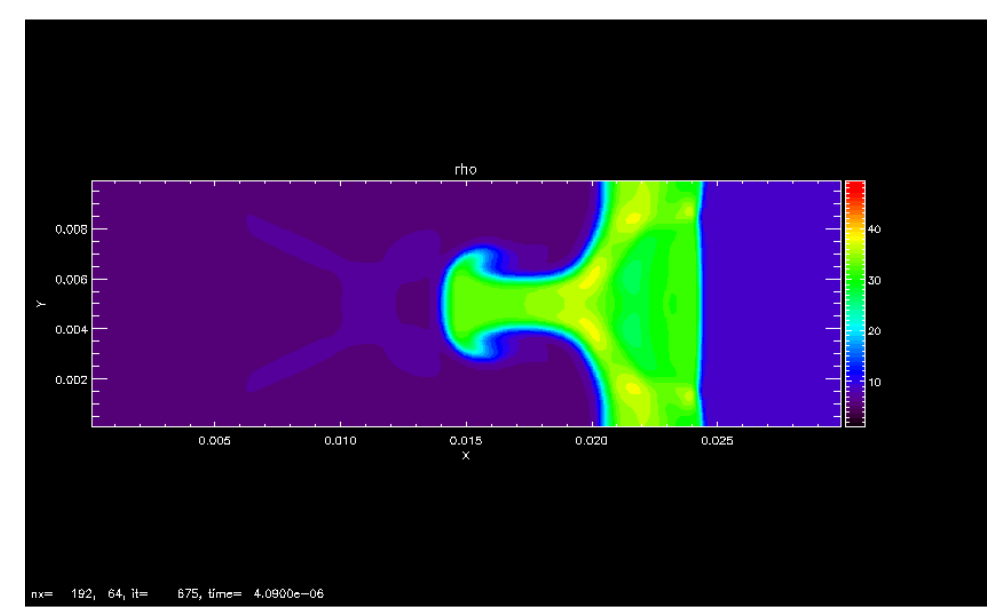

Figure 14: $C R A S H$ density plot at final time in run $M=5, A t=0.8$ and initial amplitude Amp $=0.25 \lambda$.

\section{Conclusions}

Both SPHC and CRASH codes handled the Richtmyer-Meshkov instability, managing to run all 18 simulations, and the results obtained show good agreement among the codes over the entire parameter regime and thus provide some reassurance of the quality of the numerical results. The numerical results agree qualitatively and quantitatively with the theoretical analysis, including the roll-up of vortces, development of the shear-driven Kelvin-Helmholtz instability, the oscillations of the velocity field, induced by reverberations of sound waves, dependence of the growth-rate on the amplitude of the initial perturbation, and flattening of the bubble front.

Fairly high particle resolution used in the SPHC simulations (approx. 1e5 particles) and the particle distribution optimization techniques, both allowed for the new insight in RMI dynamics. One interesting insight is the development of the Kelvin-Helmholtz instability at early stages of RMI in case of large amplitude of the initial perturbation. The other important insight, which, by the authors' knowledge, has never been reported is the 'reverse jets'. These jets are created in the roots of the spikes. They propagate in the direction opposite to the spike motion and enhance the fluid mixing. It would be interesting to conduct a study in order to experimentally check the SPHC results with the use of advanced experimental technologies [41,42].

To conclude, we found that: The nonlinear evolution of the large-scale coherent dynamics in RichtmyerMeshkov mixing is an essentially non-local and multi-scale processes. At late stages of flow evolution, the dynamics at small-scale is heterogeneous, and is characterized by the appearance of local microscopic structures. The coupling between the large and small scales has a complicated character, and appears to depart from the consideration proposed by simple heuristic models. 


\section{References}

1. Andreopoulos, Y., Agui, J.H., Briassulis, G., 2000, Shock wave-turbulence interactions, Annual Rev. Fluid Mech. 32, 309-345

2. Délery, J., Dussauge, J.-P., Some physical aspects of shock wave/boundary layer interactions Shock Waves (2009) 19:453-468

3. Dolling, D.S., 2001, Fifty Years of Shock-Wave/Boundary-Layer Interaction Research: What Next?, AIAA JOURNAL 39, 1517-1531

4. Kovasznay, L. S. G. 1953 Turbulence in supersonic flow. J. Aero. Sci. 20, 657-682.

5. Lighthill, M.J. 1953, On boundary layer upstream influence. Part I: a comparison between subsonic and supersonic flows, Proc. R. Soc. A217, 344-357; On boundary layer upstream influence. Part II: supersonic flows without separation. Proc. R. Soc. A217, 478-507

6. Ribner, H.S., 1953, Convection of a pattern of vorticity through a shock wave. NACA TN 2864

7. Ribner, H.S., 1954, Shock-turbulence interaction and the generation of noise. NACA TN 3255.

8. Ribner, H.S., 1987, Spectra of noise and amplified turbulence emanating from shock-turbulence interaction. AIAA J., 25, 436-442

9. Settles, G.S., Dodsont, L.J., 1994, Supersonic and Hypersonic Shock/Boundary-Layer Interaction Database, AIAA Journal 32, 1377-1383

10. Zel'dovich, Ya.B.,, Raizer, Yu.P., 2002, Physics of Shock Waves and High-Temperature Hydrodynamic Phenomena, Dover, New York.

11. Landau, L. D. and E.M. Lifshitz 1987b, Course of theoretical physics VI, Fluid mechanics, Pergamon Press, New-York, 1987b.

12. Abarzhi, S.I. Review on nonlinear coherent dynamics of unstable fluid interface: conservation laws and group theory, Physica Scripta T132, 297681, p1-18 (2008).

13. Abarzhi, S.I., 2010, Review of theoretical modelling approaches of Rayleigh-Taylor instabilities and turbulent mixing, Phil. Trans. Roy. Soc. A 368, Issue 1916, 1809-1828

14. Wouchuk, J.G., de Lira, C.H.R., Velikovich, A.L., 2009, Analytical linear theory for the interaction of a planar shock wave with an isotropic turbulent vorticity field, Phys Re. 79, 066315

15. Kolmogorov, A. N., 1941, "Local structure of turbulence in an incompressible fluid for very large Reynolds numbers," Dokl. Akad. Nauk. SSSR 30, 299-303; "Energy dissipation in locally isotropic turbulence," Dokl. Akad. Nauk. SSSR 32, 19-21.

16. Sreenivasan, K. R., 1999, "Fluid turbulence," Rev. Mod. Phys. 71, S383-S395.

17. Lee, S., Lele, S.K., Moin, P.,1997, Interaction of isotropic turbulence with shock waves: effect of shock strength, J. Fluid Mech. 340, 225-247

18. Mahesh, K., Lele, S.K., Moin, P., 1997, The influence of entropy fluctuations on the interaction of turbulence with a shock wave, J. Fluid Mech. 334, 353-379

19. Martin, M.P., 2007, Direct numerical simulation of hypersonic turbulent boundary layers. Part 1. Initialization and comparison with experiments, J. Fluid Mech. 570, 347-364.

20. Meshkov E E 1969 Instability of the interface of two gases accelerated by a shock Sov. Fluid. Dyn. 4101

21. Richtmyer R D 1960 Taylor instability in shock acceleration of compressible fluids Commun. Pure Appl. Math. 13297

22. Meshkov, E. E., 2006, Studies of Hydrodynamic Instabilities in Laboratory Experiments, (in Russian), Sarov, FGYC-VNIIEF, ISBN 5-9515-0069-9.

23. Rupert V 1992 Shock-interface interaction: current research on the Richtmyer-Meshkov instability in Shock Waves Proc. 18th Int. Symp. Shock Waves ed K Takayama (Berlin: Springer)

24. Drake R.P., "Perspectives of high energy density physics," Phys. Plasmas 16, 055501, 2009

25. Remington B. A., Drake R.P., Ryutov D.D., "Experimental astrophysics with high power lasers and Zpinches," Rev. Mod. Phys. 78, 755, 2006

26. O. V. Gotchev "Magneto-inertial Approach to Direct-drive Laser Fusion,” 31 J. Fusion Energy 27, 25, 2008.

27. Y.C.F. Thio et al., "A Physics Exploratory Experiment on Plasma Liner Formation,” J. Fusion Energy 20, $1,2002$.

28. J.T. Cassibry, R.J. Cortez, S.C. Hsu, F.D. Witherspoon, "Estimates of confinement time and energy gain for plasma liner driven magnetoinertial fusion using an analytic self-similar converging shock model," Physics of Plasmas 16, 112707, 2009. 
29. Wouchuk J G 2001 Growth rate of the linear Richtmyer-Meshkov instability when a shock is reflected Phys. Rev. E 63 056303; Growth rate of the Richtmyer-Meshkov instability when a rarefaction is reflected Phys. Plasmas 8 2890-907.

30. Herrmann M, Moin P and Abarzhi S I 2008 Nonlinear evolution of the Richtmyer-Meshkov instability $J$. Fluid Mech. 612 311-38

31. Kucherenko, Y. A., Shestachenko, O. E., Balabin, S. I., Pylaev, A.P., 2003, "RFNC-VNIITF multifunctional shock tube for investigating the evolution of instabilities in non-stationary gas dynamic flows," Laser Part. Beams 21, 381-384.

32. Zhakhovskii V V, Zybin S V, Abarzhi S I and Nishihara K 2006 Atomistic dynamics of RichtmyerMeshkov instability in cylindrical and planar geometries Am. Inst. Phys. Conf. Proc. 845 p $433-6$

33. Zhou Y, Remington BA, Robey HF, Cook AW, Glendinning SG, Dimits A, Buckingham AC, Zimmerman $\underline{\mathrm{GB}}, \underline{B u r k e} \mathrm{EW}, \underline{\text { Peyser TA}}, \underline{\text { Cabot } \mathrm{W}}$, Eliason D Progress in understanding turbulent mixing induced by Rayleigh-Taylor and Richtmyer-Meshkov instabilities Physics of Plasmas 10, 1883-1896, 2003.

34. Aglitskiy Y, Velikovich A L, Karasik M, Serlin V, Pawley C J, Schmitt A J, Obenschain S P, Mostovych A N, Gardner J H and Metzler N 2001 Direct observation of feedout-related mass oscillations in plastic targets Phys. Rev. Lett. 87265002

35. Aleshin A N, Lazareva E V, Zaytsev S G, Rozanov V B, Gamalii E G and Lebo I G 1990 Linear, nonlinear and transitional stages in the development of the Richtmyer-Meshkov instability Sov. Phys. Dokl. 35159.

36. Velikovich A L and Dimonte G 1996 Nonlinear perturbation theory of the incompressible RichtmyerMeshkov instability Phys. Rev. Lett. 76 3112-3115.

37. Brouillette M 2002 The Richtmyer-Meshkov instability Ann. Rev. Fluid Mech. 34 445-68

38. Holmes R L, Dimonte G, Fryxell B, Gittings M L, Grove J W, Schneider M, Sharp D H, Velikovich A L, Weaver R P and Zhang Q 1999 Richtmyer-Meshkov instability growth: experiment, simulation and theory J. Fluid Mech. 389 55-79

39. Abarzhi S I 2002 A new type of the evolution of the bubble front in the Richtmyer-Meshkov instability Phys. Lett. A 294 95-100.

40. Abarzhi S I, Nishihara K and Glimm J 2003 Rayleigh-Taylor and Richtmyer-Meshkov instabilities for fluids with a finite density ratio Phys. Lett. A 317470.

41. Orlov, S.S., Abarzhi, S.I., Barbastathis, G., Se-Baek Oh, Sreenivasan, K.R., "New technologies for fluid dynamic experiments and diagnostics," Philosophical Transactions of the Royal Society A, in preparation (2009).

42. Abarzhi S.I. 2010, On fundamentals of Rayleigh-Taylor turbulent mixing, Europhysics Letters 91, 12867.

43. Liu, G.R., Liu, M.B., "Smoothed Particle Hydrodynamics - a meshfree particle method". 2003, New Jersey, London, Singapore, Shanghai, Hong Kong, Taipei,Bangalore: World Scientific. 449.

44. Monaghan, J.J., Gingold, R.A., "Shock simulation by the particle method SPH". Journal of Computational Physics, 1983(52): p. 374-389.

45. Monaghan, J.J., Poinracic, J., “Artificial viscosity for particle methods”. Applied Numerical Mathematics, 1985. 1: p. 187-194.

46. Monaghan, J.J., "SPH meets the Shocks of Noh". Monash University Paper, 1987.

47. Monaghan, J.J., “An introduction to SPH”. Computer Physics Communications, 1988. 48: p. 89-96.

48. Monaghan, J.J., "Smoothed Particle Hydrodynamics". Reports on Progress in Physics, 2005. 68: p. 1703-1759.

49. B. Einfeldt, C. D. Munz, P. L. Roe, and B. Sjgreen. "On godunov-type methods near low densities". Journal of Computational Physics, 92(2):273 - 295, 1991.

50. Moylan, B.E., "Raindrop demise in high-speed projectile flowfield", Ph.D. dissertation, Mechanical and Aerospace Dept., University of Alabama in Huntsville, AL, 2010.

51. Chandrasekhar, S., Hydrodynamic and hydromagnetic stability, Oxford University Press, 1961.

52. Joseph, D.D., Stability of Fluid Motions Vol 2, Springer Verlag, 1976

53. Sokolov I.V., Hydrodynamic cumulative processes in plasma physics, Uspekhi Fizicheskikh Nauk 160, Issue $11,143-166,1990$. 